\title{
Research on Employment Information Management Platform for Colleges and Universities Based on Big Data
}

\author{
Meiqin Li \\ Department of Information Engineering, Shandong Vocational College of Science and Technology, Weifang, Shandong Province, \\ 261053, China
}

\begin{abstract}
It is an era of big data nowadays. Processing, analyzing and working with data are new opportunities and challenges. This article mainly studies the development and application of employment information management platform of colleges and universities under the background of big data. As the number of graduates increases year by year, the employment situation is becoming more and more severe. The employment information management platform is playing an increasingly important role as an information exchange platform for colleges and universities to learn about the employment situation of students, and for employers to release recruitment information and for students' job hunting. The employment information management platform makes full use of big data for analysis, which can well control the employment; at the same time, the school grasps the changing trend of society's needs for talents through analysis, and the employment situation of graduates will be used as the main basis to revise talent training plans, formulate enrollment plans, and carry out feasibility study for adding new majors. The big data platform provides a basis for decision-making of colleges and universities and is of great significance to the development of school. Therefore, it is extremely urgent to build an efficient and convenient employment information management platform to meet the needs of colleges and universities, employers and students. Keywords - big data, data mining, employment information platform, three-tier frame
\end{abstract}

\section{Introduction}

In recent years, the number of college graduates in China has increased year by year. According to the Ministry of education, the number of graduates in 2020 will reach 8.74 million, the highest in history. However, influenced by COVID-19, the overall growth rate and volume of the national economy have been greatly affected. Correspondingly, the employment market situation is not optimistic, and the employment market for graduates is the first to bear the brunt.

Entering the 1990s, the computer technology has developed rapidly. Correspondingly, network and communication technology also advanced by leaps and bounds, penetrating and affecting all walks of life. From a global perspective, some western capitalist countries have significant advantages in this regard, and their theoretical and practical levels have reached a higher level. The various information management platforms they designed have formed a relatively mature system in terms of frame design and function expansion. In terms of employment management, the general employment information statistics management platform for college and university graduates has been basically realized. The building of related networks and software research are also commonly found in these countries, and all networked information sharing mechanisms have been realized, which have provided an important boost for social and economic development.

In recent years, the employment of domestic college and university graduates has also developed rapidly, and tremendous changes have also taken place in the employment guidance methods. Most colleges and universities have established relatively complete employment information management platforms and have achieved certain results. However, in terms of platform building and other aspects, the level is still uneven, and there are some existing problems, among which the followings are more representative: for example, insufficient functions of website, which are not suitable for multi-level and diversified needs, and most college websites have a single function and are mainly developed around the release of graduates and employers' information; the accuracy and authenticity of some information cannot be guaranteed; some websites are not high in informationalized level; insufficient utilization rates showed that they have not really played their role. The development and utilization of this type of information resources often requires a large amount of manpower, material, and financial resources. At the same time, the building of corresponding management teams, mechanisms and systems is also very important. Especially at present, many websites do not have specialized management teams, making the website useless and causing a great waste. 


\section{Formatting the title, authors and affiliations}

\subsection{Demand analysis of employment information platform}

The first step in developing a management platform is demand analysis, the purpose of which is to establish a communication bridge between platform developers and users. The use by the relevant users is the ultimate goal of platform design. The developers should extensively collect opinions and conduct targeted analysis before platform design. Otherwise, the practicality of the platform developed cannot be guaranteed.

The employment information management platform for graduates requires a relatively simple working environment. The actual needs of college and university employment work require that the platform design must be guided by it. Through the preliminary research, the majority opinions of several students, college teachers, and corporate HR leaders have been synthesized to form a more detailed research report, which clarifies each function required by the platform. According to the research, the users of the platform can be roughly divided into the following three categories: college and university graduates, employment management staff and corporate users. Different users have different requirements for platform functions, so the tasks that platforms need to complete are also different.

The performance requirement analysis is to ensure that a more reasonable information management platform is designed, which can adapt to the characteristics of networked management, enhance the stability of the platform, and extend the use time. In the actual operation process, all corporate and student information closely related to the entire system should be kept confidential. According to relevant laws and regulations, leaking graduates' personal information is an act of infringing on the privacy of others. Therefore, security is very important to this platform. Security should be considered from a wide range of perspectives, which can be divided into three aspects: application level, user level and platform level.

In short, centralized management, friendly interface and large-scale data processing capabilities are in line with the three-tier structure of the $\mathrm{B} / \mathrm{S}$ mode combination. It is the most basic design requirement of the employment information management platform, which can maximize the informatization needs of the employment of college and university graduates.

\subsection{Overall design of employment information platform}

This platform adopts the $\mathrm{B} / \mathrm{S}$ mode, which is not restricted by space and time. As long as the server is running normally, users can access the platform at any time or place (through successful connection of PC to the Internet). The Platform Frame Diagram is shown in Figure 1 below.

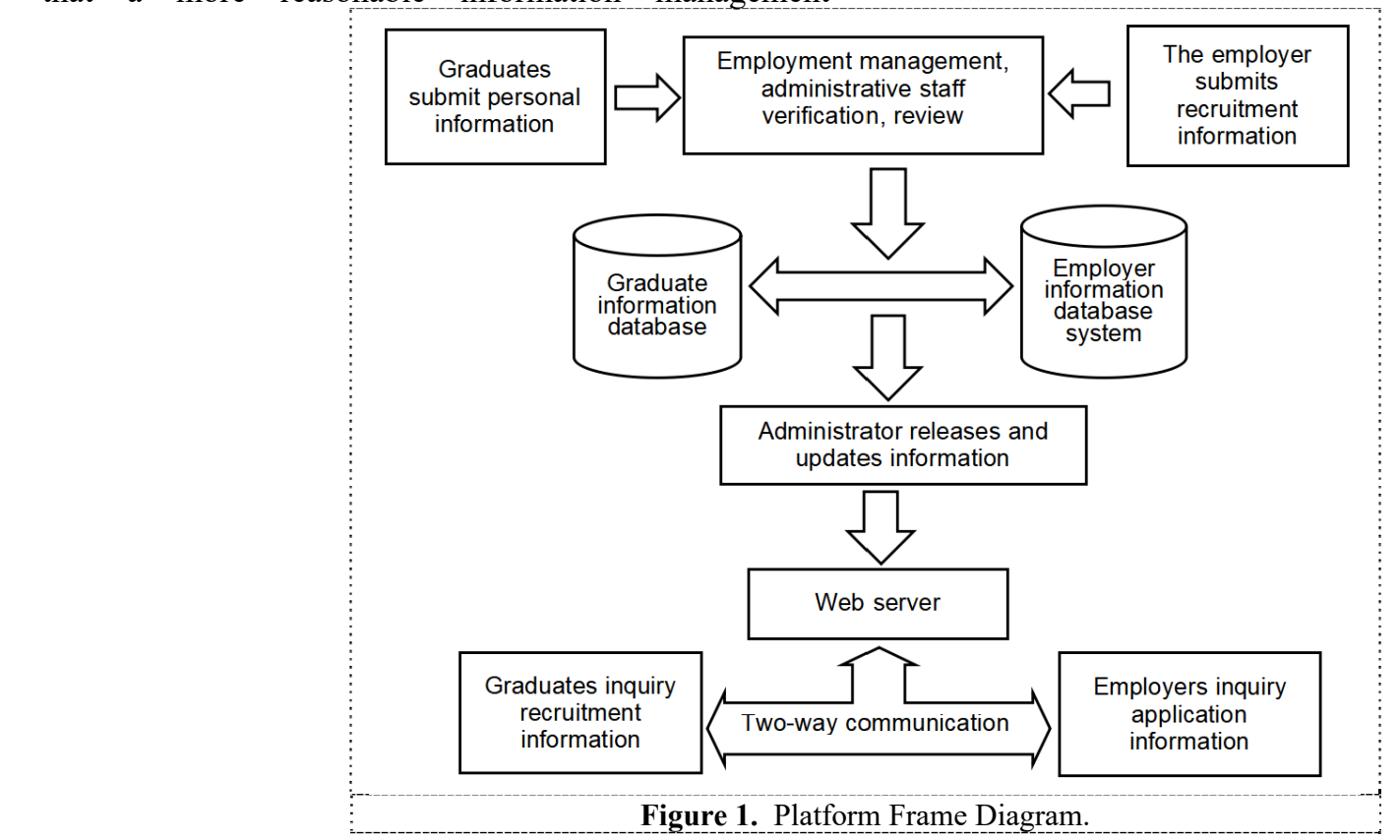

\subsection{Database design of employment information platform}

The core of database design is the design of logical and physical databases, which is also the basic requirement of standardized design methods. The design of the logical database, in accordance with the relevant database design theory, refines the step-by-step and partial logic on the basis of determining the overall logic, in addition to combining the characteristics of the database itself and the specific and detailed requirements of the user. The physical design is relatively simple. After the logical structure is determined, the storage structure of the database and other details are designed. The logical structure of the database is inseparable from the database model. Data objects, properties of various objects, and relationships between objects are the three 
main characteristics of the logical structure of a database. This data description is very classic.

There are three types of platform users in this platform: student users, enterprise users, and administrators. The design of each entity in the platform and its corresponding properties will have a direct impact on the description of the platform model, so it is necessary to draw and analyze one by one. The entities included in this platform are relatively standardized: students, enterprises, administrators, job hunting information, job recruitment entities, etc. After drawing the specific entities from the platform, the E-R model can be established based on the relationship data between these entities. The relationship between entities can be divided into one-to-one relationships, one-to-many relationships, and many-to-many relationships. For example, the relationship between student entities and job hunting information entities is a many-to-many relationship, that is, any student can issue many job hunting posts.

\subsection{Employment information platform test}

Discovering the possible errors is the main purpose of our software testing. Being able to find the undiscovered errors is the most successful proof of a software test. An indispensable part of the test data preparation is the preinformation output. It is a method that programmers often use to write the pre-output of the program and compare it with the actual output result, so that it is easier to find potential errors in the program. Therefore, a detailed description of the program input data and a detailed explanation of the program anticipated output results constitute a complete test data.

This platform has passed multiple strict tests and fully meets the requirements in terms of grammar and computer execution. The introduction of the part of computer debugging of this article is based on the employment information management platform as an example. The test consists of three parts, which are divided into user interface test, basic function test, and logic algorithm test. The test results show that it can perfectly realize the function of data report statistics; the platform itself has strong fault tolerance and can partially self-repair faults and correct errors.

\section{Realization of key technologies}

\subsection{AJAX technology}

AJAX is a new technology composed of several technologies, which describes two powerful features of JavaScript: it can automatically send the same command to the remote Serve without the user's refresh instruction; analysis and process XML files. It is required to write server-side and client-side programs to develop AJAX applications. The following two steps will illustrate the application and implementation of AJAX technology in this project.

- $\quad$ Send an HTTP request to the server
First, an HTTP request is sent to the server by JavaScript. At this time, a class sample is required, and the sample must have this function. A cross-browser sample will be created in this way, the code used is as follows:

if(window. XMLHttpRequest) \{

http_request=new XMLHttpRequest();

\}else if(window. ActiveXObject)\{

http_request $=$ new

ActiveXObject("Microsoft. XMLHTTP"); \}

- The response of the processor

Providing the JavaScript function name of the specified response is a prerequisite for sending the request.

http_request. onreadystatechange=nameOfTheFunction;

After entering the program sequence, the platform will automatically check the state of the variable and provide a return value. If the return value is 4 , it means that a complete server response has been completed. After the user commands and the status values of the HTTP server are checked one by one, we have the right to access and download the data of the remote Serve. There are two main ways to get these data:

http_request. responseText-receive data as a text string

http_request. responseXML-If the message provided by the server contains an XML document, it will be returned in XML format.

\subsection{Crawler technology}

Web crawler is a script that extracts web page data according to certain rules, which is widely used in Internet search engines. It can automatically collect all the accessible page content to obtain or update the content and retrieval methods of these websites.

\#Function definition, data capture

def get_urlpage(url):

$\mathrm{ht}=$ get_html(url)

$\mathrm{cn}=$ re. findall $\left(\mathrm{r}^{\prime}<\operatorname{div}\right.$ class $=" \mathrm{cn} ">(.+$ ? $)<$ $/$ div $>$ ', ht, re. I|re. M|re. S)

for $\mathrm{i}$ in soup.find_all(lambda tag: tag.name $=={ }^{\prime} \mathrm{div}^{\prime}$ and tag.get('class') $\left.==\left[{ }^{\prime} \mathrm{el} '\right]\right)[4:]$ :

job $=$ i.find $(' p$ ',class_='t1').a['title']

company $=$ i.find('span',class $=$ 't2').a['title']

place $=$ i.find('span',class_ $=$ 't $3^{\prime}$ ').get_text( $)$

salary $=$ i.find('span',class_ $=$ 't4').get_text()

date $=$ i.find('span', class_='t5').get_text()

detail_url = i.find ('p',class_='t1').a['href']

with open('intro job.csv', 'a+', encoding='utf-8-sig') as f: f.write(job + ',' + company + ',' + place + ',' + salary + ',' + date +',' + detail_url + 'n') \#Data crawling

\section{Conclusion}

China has entered an information age with rapidly developing speed. The employment information management platform is playing an increasingly important role as an information exchange platform for colleges and universities to learn the employment 
situation of students, and for employers to release recruitment information and for students to have job hunting. Based on the principle of starting from actual needs, using platform-based and standardized design methods, the development of college and university employment information management platform has been realized.

The management of the employment by colleges and universities is realized through the student employment information management platform, which provides a convenient platform for job hunting by students and recruitment of talents by enterprises. According to different users, the whole platform is divided into three sub-platforms: student management sub-platform, enterprise management sub-platform and platform management sub-platform. Among them, the student management sub-platform mainly covers the function of the maintenance of basic student information, the releasing of job search information, the maintenance of personal resumes, and online communication, etc; the enterprise management sub-platform mainly provides basic corporate information maintenance, releasing recruitment information, talents-searching, and online communication, etc. The platform management subplatform is mainly for the background operation of the entire platform, including the setting of user rights and the maintenance of the background database. Specifically, it covers functions such as user information management, employment information management, and database maintenance.

Although the employment information management platform has basically completed its main functions, with the rapid development of information technology, the data in the database is becoming larger and the information is becoming more complex, higher requirements are put forward for the improvement of the platform in the future.

\section{Acknowledgments}

On the occasion of the completion of this paper, I would like to thank the relevant leaders and colleagues for their strong support and cooperation, especially during the period of data collection, collation and system construction, which has been helped by many technical personnel. I would like to express my thanks.

In addition, I would like to express my gratitude to my family for your support in my work and study.

\section{References}

1. Vocational Technology. Jia Tianxia. Research on Personalized Employment Guidance in Higher Vocational Colleges Based on Employment Information Platform[J].2018, 17(4): 32-34.

2. Science and Technology Information.Qin Dongmei, Zhong Shouguang, Guan Peng etc. Development of College Employment Information Integration System under the Background of Big Data[J]. 2017, 15(17): 15-16.
3. Modern Communication.Zhu Nan. Research on the Application of the Employment Information Management System for Domestic College Students[J]. 2018(3): 35-36.

4. Education and Teaching Forum. Liang Ben. On the Application of Big Data Thinking in Employment Guidance in Colleges and Universities[J]. 2019(49)

5. Modern Electronic Technology. Li Xiaohong, Chang Zhenyun. Fuzzy Improved Clustering Algorithm of Data Mining Model in Big Data[J]. 2020(03)

6. Computer and Modernization. Li Jian, Yang Xing, Li Juncheng, etc. The Design of Employment Information Management Platform Based on Big Data Technology and Feature Recommendation[J]. 2018(06).

7. Computer Knowledge and Technology. Zhang Siyang, Huang Tongcheng. Exploration and Application of Big Data in Employment Management in Colleges and Universities [J]. 2019(10)

8. Modern Information Technology. Ni Qianlong. Research on the Construction of University Employment Information Service Platform Based on Big Data[J]. 2019(19) 